\title{
In situ Encapsulation of $E$. coli in GLC and Prediction of Beam Induced Death
}

David J. Banner ${ }^{1}$, Justas Jakubonis ${ }^{1}$, Emre Firlar ${ }^{1,2}$, Jodi K. Finlay ${ }^{1}$, Agata Bogdanowicz ${ }^{1}$, Reza Shahbazian Yassar $^{2}$, Constantine Megaridis ${ }^{2}$, Tolou Shokuhfar ${ }^{1,2}$

1. University of Illinois at Chicago, Department of Bioengineering, Chicago, IL, USA

2. University of Illinois at Chicago, Department of Mechanical and Industrial Engineering, Chicago, IL, USA

Previous in situ electron microscopy works have encapsulated bacteria within liquid flow holders and demonstrated viability of bacteria under low dose electron beam exposure, but have so far been limited to $5 \mathrm{~nm}$ resolution due to the thickness of the silicon nitride windows of liquid flow holders and the increased liquid thickness [1]. Encapsulation of a wet sample between graphene sheets, graphene liquid cells (GLCs), previously achieved atomic resolution imaging of biological samples, but is often thought to not allow encapsulation of samples larger than several hundred nanometers. However, encapsulation of the thick-cell walled gram-positive $B$. subtillis has previously been shown [2]. Herein, gram-negative E. coli, with a cell wall thinner than B. subtillis, are encapsulated within GLCs. E. coli are rod-shaped cylindrical bacteria and are typically $500 \mathrm{~nm}$ to $1 \mu \mathrm{m}$ in diameter and $1 \mu \mathrm{m}$ to $3 \mu \mathrm{m}$ in length. This encapsulation shows that: 1) GLC may be used to encapsulate samples much larger than the often perceived 200nm limit, 2) E. coli and other gram-negative bacteria may be encapsulated within GLC to observe real-time phenomena at the nanoscale, 3) morphological changes can be used to identify the $E$. coli death within GLC, and 4) these morphological changes can be predicted to anticipate the death of the bacterium under known electron doses. Herein, the electron dose was varied to visualize the live state and the real-time death of the bacterium.

The live/dead state of bacteria, both in general and of E. coli in particular, is determined by the integrity of the bacterium's cell wall for two excellent reasons: If the bacterium's cell wall is structurally damaged, the bacterium dies. Alternatively, if the cellular processes of the bacterium are disrupted and the bacterium dies as a result, the cell wall is damaged and reflects the prior death of the bacterium. At the micrometer scale, this phenomenon is typically detected by fluorescent live/dead staining rather than by direct imaging facilitated by higher resolution electron microscopy. Here, E. coli glycerol stocks preserved at $-80^{\circ} \mathrm{C}$ were used to inoculate LB broth cultures. E. coli cultures were incubated at $37.5^{\circ} \mathrm{C}$ for 12 hours to 24 hours. Samples were centrifuged to remove the LB broth and the bacteria rehydrated in phosphate buffer saline (PBS). A sample of this solution was encapsulated in GLC for electron microscopy, while a viability of $85 \%$ of the solution was simultaneously determined via live/dead fluorescent staining. The GLC imaging and sample behavior within the electron microscope was videorecorded.

In Figure 1A, ex situ E. coli, not encapsulated in GLC, exhibited highly damaged cell morphology and cell death in Scanning Transmission Electron Microscope (STEM). The localization of the STEM electron beam ensures that unviewed areas endure minimal electron doses, indicating that widespread bacterial death is due to the low-pressure vacuum and the dehydration of the sample. In contrast, an $80 \mathrm{kv}$ Transmission Electron Microscope (TEM) was used with a small aperture and low magnification to locate the in situ bacterium within GLC shown in Figure 1B and 1C. As can be seen in Figure 1B, the bacterium cell wall appears undamaged after brief low-dose beam exposure, not showing any visible separation of the cell wall or uneven, damaged cell morphology. This confirms the bacterium is fully 
encapsulated by graphene and likely survived GLC encapsulation. After collection of Figure 1B, the small aperture was removed to allow higher dose beam exposure. During the experiment, the area directly around the bacterium and in the lower right corner exhibited bubbling and gas production due to radiolysis induced by the electron beam exposure. This bubbling confirms the presence of encapsulated liquid. In Figure 1C, the smaller, damaged bacterium is shown after 240 seconds of high beam electron beam exposure, exhibiting both a reduction of volume due to escape of the cytosol from the cell and separation of the cell wall from the cell body (Figure 1C8). This change in volume was modeled by $V=$ $\frac{V_{B 0}+\alpha_{1} e^{\beta t}}{1+\alpha_{2} e^{\beta t}}$, where $V$ is the volume, $V_{B 0}$ the initial volume, $\alpha_{1}$ and $\alpha_{2}$ permeability constants of the membrane, while $\beta$ reflects the rate of volume change [3]. The longevity of bacteria under a known electron dose may then be predicted by determining $\alpha_{1}, \alpha_{2}$, and $\beta$. In Figure 1D, the bacterium volume loss is plotted to show the reduction of the bacterium volume due to the escape of cytosol from the compromised cell wall, from which $\alpha_{1}, \alpha_{2}$, and $\beta$ may be derived.

While direct fluorescent live/dead staining of bacteria remains difficult due to the technical difficulty of locating the same bacterium before and after electron microscopy, the lack of morphological changes within GLC samples indicate that the majority of $E$. coli survive encapsulation. This survival indicates that the primary consideration of GLC of gram-negative bacteria is the electron beam dose. In addition to the higher resolution of GLC as compared to liquid flow holders, the decreased liquid thickness and the permeability of graphene to the electron beam produces fewer secondary electrons, which lowers the total electron dose experienced by the bacteria [4].

\section{References:}

[1] E Kennedy et al, ACS Nano. 10 (2016), p. 2669.

[2] J Park et al, Nano Lett. 15 (2015), p. 4737.

[3] N Mohanty et al, Nano Lett. 11 (2011), p. 1270.

[4] The authors gratefully acknowledge Nonwovens Institute award \#16-195, NSF award \#1710049, and NSF CAREER award \#1564950
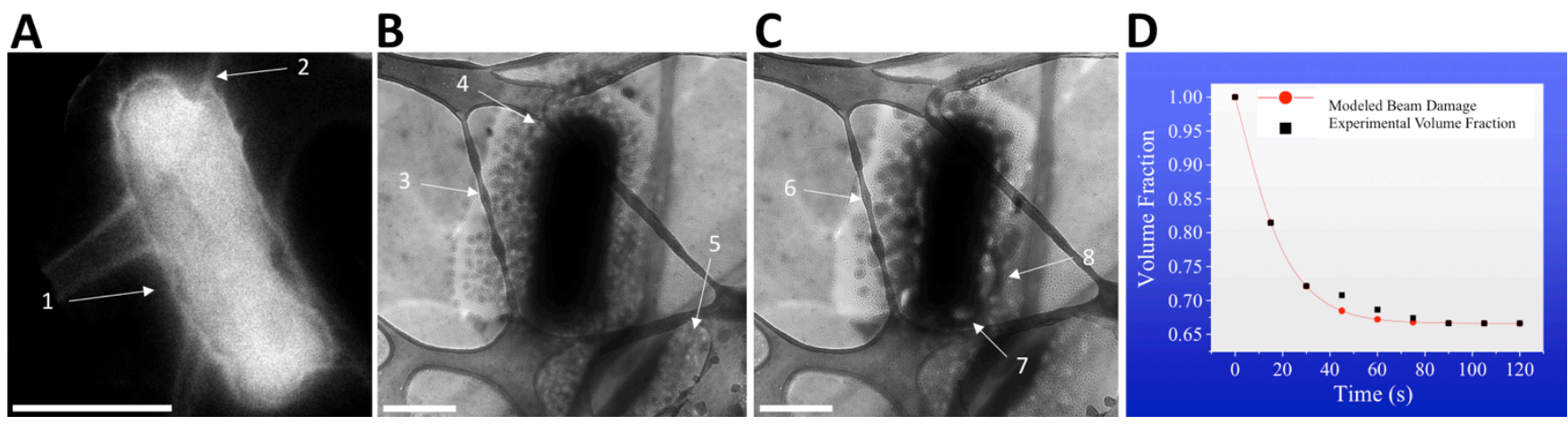

Figure 1. All scale bars are 500nm. In A, E. coli is imaged ex situ in Scanning Transmission Electron Microscopy at 200kV. A1 indicates the roughening of the cell wall and loss of the smooth cell wall morphology associated with cellular death. A2 indicates a complete break in the cell wall. In B and C, E. coli is encapsulated in GLC in Transmission Electron Microscopy at 80kV. In B, the image is immediately recorded upon beam exposure. B3 indicates salts from the PBS medium. B4 indicates the smooth, intact cell wall. B5 shows a thicker liquid within the cell bubbling. In C, the image was collected after 240 seconds of high electron dose exposure. In C6, the salts have aggregated due to electron beam induced reactions. C7 indicates the completely broken cell wall of the bacterium, similar to A2. C8 shows the cell wall, observed to slowly detach from the bacterium throughout the beam exposure. In D, the reduction in volume of the bacteria is modeled. Black squares are the experimentally measured volume fractions, while the red line and red circles represent the model predicted graph. 